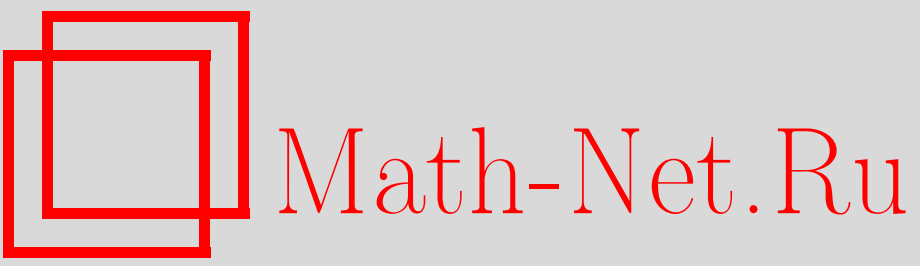

Б. А. Арбузов, Спонтанное возникновение эффективного взаимодействия в ренормируемой модели квантовой теории поля, ТМФ, 2004, том 140, номер 3, $367-$ 387

DOI: https://doi.org/10.4213/tmf99

Использование Общероссийского математического портала Math-Net.Ru подразумевает, что вы прочитали и согласны с пользовательским соглашением http://www . mathnet.ru/rus/agreement

Параметры загрузки:

IP: 54.198 .55 .26

26 апреля 2023 г., $15: 43: 02$ 
ТЕОРЕТИЧЕСКАЯ

И МАТЕМАТИЧЕСКАЯ

ФИЗИКА

Том 140, № 3

сентябрь, 2004

(C) 2004 г.

Б. А. Арбузов*

\section{СПОНТАННОЕ ВОЗНИКНОВЕНИЕ ЭФФЕКТИВНОГО ВЗАИМОДЕЙСТВИЯ В РЕНОРМИРУЕМОЙ МОДЕЛИ КВАНТОВОЙ ТЕОРИИ ПОЛЯ}

\footnotetext{
Рассмотрена теория безмассового скалярного поля $\phi$ с взаимодействием $g \phi^{3}$ в шестимерном пространстве. С применением метода квазисредних Боголюбова изучена возможность нарушения исходной масштабной инвариантности, связанная со спонтанным возникновением эффективного взаимодействия $G \phi^{4}$. Показано, что линеаризованное уравнение компенсации для формфактора этого взаимодействия в приближении до третьего порядка включительно имеет нетривиальное решение. В этом же приближении имеет решение также уравнение Бете-Солпитера для безмассового скалярного связанного состояния двух полей $\phi$. Согласование значения формффактора в нуле и массы скалярного поля $m$ приводит к однозначному решению, которое дает связь между параметрами взаимодействия $g \phi^{3}$ и параметрами $G$ и $m$. Приведены аргументы в пользу устойчивости полученного нетривиального решения.
}

Ключевые слова: эффективное взаимодействие, квантовая теория поля, подход квазисредних Боголюбова, уравнение компенсации, нетривиальное решение.

\section{1. УРАВНЕНИЕ КОМПЕНСАЦИИ В МЕТОДЕ КВАЗИСРЕДНИХ}

Наиболее последовательным и продуктивным методом исследования проблемы спонтанного нарушения симметрии является метод квазисредних Боголюбова [1], [2]. Важнейшей составной частью этого метода является уравнение компенсации. Кратко сформулируем способ получения уравнений компенсации.

При изучении возможности спонтанного нарушения симметрии в задачах квантовой теории поля в методе квазисредних [2] производится следуюшая процедура ${ }^{1)}$. Пусть имеется исходный лагранжиан

$$
L=L_{0}+L_{\mathrm{int}},
$$

1) Впервые методы работ [1], [2] были применены к задачам квантовой теории поля в работе [3].

*Научно-исследовательский институт ядерной физики им. Д. В. Скобельцына, Московский государственный университет, Москва, Россия. E-mail: arbuzov@theory.sinp.msu.ru 
обладающий некоторой симметрией. Добавим к выражению (1) член $\epsilon L_{\mathrm{br}}$, нарушаюший исходную симметрию. В модифицированной таким образом задаче проводится вычисление интересующих нас величин и переход к пределу $\epsilon \rightarrow 0$ осуществляется только после этих выгислений. Далеко не всегда результаты такой процедуры (квазисредние) совпадают с результатами, полученными в рамках исходной симметричной постановки задачи (просто средними). При этом в процессе вычисления квазисредних необходимо решать уравнение компенсации. Например, в исходной теории с киральной симметрией фермионы обязаны иметь нулевые массы. Используем в качестве нарушающей малой добавки

$$
\epsilon L_{\mathrm{br}}=-\epsilon \bar{\psi} \psi
$$

Теперь добавим к модифицированному лагранжиану и вычтем из него возможный массовьп̆ член,

$$
L=L_{0}-m \bar{\psi} \psi+L_{\mathrm{int}}+m \bar{\psi} \psi-\epsilon \bar{\psi} \psi
$$

Объединим два первых члена в новый свободный лагранжиан, а три последних - в новый лагранжиан взаимодействия. При этом мы должны потребовать, чтобы новое взаимодействие не давало вклада в массовый член, т.е. чтобы двухчастичная функция Грина, полученная с помощью модифицированного лагранжиана взаимодействия, на массовой поверхности обрашалась в нуль. Это требование и есть уравнение компенсации. В рассматриваемом случае это требование приводит к уравнению

$$
-m+\epsilon+\Sigma(m)=0
$$

где $\Sigma(m)$ - массовый оператор на массовой поверхности модифицированного свободного лагранжиана. В этом уравнении уже можно выполнить предельный переход $\epsilon \rightarrow 0$. Как правило [3], массовый оператор $\Sigma(m)$ пропорционален $m$, так что всегда существует тривиальное решение уравнения компенсации $m=0$. Однако может сушествовать и нетривиальное решение $m \neq 0$.

Итак, основным принципом построения уравнения компенсации является процедура “добавить - вычесть” и отнесение одного нарушаюшего симметрию члена в свободный лагранжиан, а другого - в лагранжиан взаимодействия. Затем нужно скомпенсировать тот член, которьй должен обращаться в нуль в рассматриваемой задаче. Этот принцип мы и используем в настояшей работе.

\section{2. ОБОСНОВАНИЕ ВЫБОРА МОДЕЛИ}

Явление спонтанного нарушения инвариантности играет решаюшую роль в формулировке электрослабой теории. Как известно, стандартный способ нарушения требует введения элементарных скалярных полей [4], самодействие которых приводит к перестройке вакуума теории и к предсказанию существования скалярной хиггсовой частишы. Наряду со стандартным подходом рассматриваются и предложения, основанные 
на динамическом нарушении исходной симметрии без элементарных хиггсовых скаляров. Эти возможности приводят к постулированию (см., например, обзор [5]) или к динамическому возникновению [6], [7] эффективных четырехфермионных взаимодействий тяжелых кварков. В результате исходная теория, содержашая наряду со свободным лагранжианом также и калибровочные масштабно-инвариантные взаимодействия, преобразуется в теорию, содержашую взаимодействия с размерной константой связи и явно нарушаюшую масштабную инвариантность. В работах [6], [7] сделано предположение о сушествовании решений соответствуюших уравнений компенсации, однако это предположение надлежашим образом не обосновано. Целью данной работы является изучение более простой модели, в которой удалось бы на основе точных решений (приближенных) уравнений компенсации получить условия выполнения сделанных предположений. При этом модель должна в какой-то степени соответствовать реалиям электрослабой теории. Для простоты рассматривается одно скалярное поле. Чтобы константы взаимодействий имели нужные размерности, мы выбираем размерность пространства-времени, равную шести. Действительно, тогда константа взаимодействия $g \phi^{3}$ безразмерна, а константа взаимодействия $G \phi^{4}$ имеет размерность обратного квадрата массы (в соответствии с размерностью константы четырехфермионного взаимодействия четырехмерного пространства).

Итак, в пространстве размерности шесть вводим скалярное поле $\phi$ с исходным масштабно-инвариантным лагранжианом

$$
L=\frac{1}{2} g^{\mu \nu} \frac{\partial \phi}{\partial x^{\mu}} \frac{\partial \phi}{\partial x^{\nu}}+\frac{g_{0}}{3 !} \phi^{3} .
$$

Выбираем естественную сигнатуру с одним временем и пятью пространственными осями. В этом случае переход от исходного пространства к евклидовому шестимерному сопровождается заменами

$$
p^{2} \rightarrow-p_{\mathrm{E}}^{2}, \quad d^{6} p \rightarrow i d_{\mathrm{E}}^{6} p .
$$

Для нас было важно найти хотя бы одну одну модель, которая иллюстрирует предлагаемый подход. В настоящей работе мы не ставим себе целью обсуждать физический смысл многомерной теории. Мы рассматриваем выбранньй вариант лишь как модельньй, аналогично тому, как рассматривают двумерные модели.

Итак, мы исходим из лагранжиана (5). Проводя очевидные выкладки, получаем однопетлевое уравнение ренормализационной группы [8] для бегушей константы связи $g^{2}\left(\mu^{2}\right)$,

$$
\frac{d g^{2}\left(\mu^{2}\right)}{d M}=-\frac{3 g^{4}}{4(4 \pi)^{3}}, \quad M=\ln \frac{\mu^{2}}{\Lambda_{3}^{2}},
$$

где $\Lambda_{3}$ - стандартный параметр. Решение уравнения (7) имеет вид

$$
g^{2}\left(\mu^{2}\right)=g_{0}^{2}\left(1+\frac{3 g_{0}^{2}}{4(4 \pi)^{3}} \ln \frac{\mu^{2}}{\Lambda_{3}^{2}}\right)^{-1} .
$$


Иногда удобно использовать параметр $\bar{h}\left(\mu^{2}\right)$, определенный соотношением

$$
\bar{h}\left(\mu^{2}\right)=\frac{3 g^{2}\left(\mu^{2}\right)}{4(4 \pi)^{3}}=\left(\ln \frac{\mu^{2}}{\Lambda_{g}^{2}}\right)^{-1}
$$

где при переходе от $\Lambda_{3}^{2}$ к $\Lambda_{g}^{2}$ мы использовали стандартный прием, аналогичный используемому в квантовой хромодинамике,

$$
\Lambda_{g}^{2}=\Lambda_{3}^{2} \exp \left(-\frac{4(4 \pi)^{3}}{3 g_{0}^{2}}\right)
$$

Мы убедились, что теория с лагранжианом (5) является асимптотически свободной, а выражение (9) имеет смысл при $\mu^{2} \gg \Lambda_{g}^{2}$.

Отметим, что в рассматриваемой модели имеются квадратичные расходимости в массе скалярного поля. Это общая черта теорий с элементарными скалярами. Подробнее вопрос о массе скалярного поля будет рассмотрен ниже.

\section{3. УРАВНЕНИЕ КОМПЕНСАЦИИ В ШЕСТИМЕРНОЙ СКАЛЯРНОЙ МОДЕЛИ}

Итак, мы имеем безмассовое скалярное поле шестимерного пространства. Исходный лагранжиан (5) обладает масштабной симметрией. Будем искать решение, нарушающее эту симметрию, с помощью метода квазисредних [2]. Добавим к лагранжиану мальй член $-\epsilon \phi^{4} / 4$ !. Теперь масштабная инвариантность будет нарушена, и возможно появление, вообше говоря, нелокальных членов вида

$$
G \int \bar{F}\left(x, x_{1}, x_{2}, x_{3}, x_{4}\right) \phi\left(x_{1}\right) \phi\left(x_{2}\right) \phi\left(x_{3}\right) \phi\left(x_{4}\right) d x_{1} d x_{2} d x_{3} d x_{4}
$$

где $G$ - размерная константа связи, а $\bar{F}\left(x, x_{1}, x_{2}, x_{3}, x_{4}\right)$ есть функция четырех разностей координат $x-x_{i}, i=1,2,3,4$, фурье-образ которой $F\left(p_{1}, p_{2}, p_{3}, p_{4}\right)$, где $p_{i}-$ импульсы концов, имеет смысл форм-фактора, определяющего область действия взаимодействия (10). Будем искать решение, убываюшее на бесконечности по импульсам и, стало быть, определяющее область эффективного действия рассматриваемого взаимодействия.

Представим исходньй лагранжиан (5) с малой добавкой в виде

$$
L=\frac{1}{2} g^{\mu \nu} \frac{\partial \phi}{\partial x^{\mu}} \frac{\partial \phi}{\partial x^{\nu}}-\frac{m^{2}}{2} \phi^{2}-\frac{G}{4 !} F \cdot \phi^{4}-\frac{\epsilon}{4 !} \phi^{4}+\frac{g_{0}}{3 !} \phi^{3}+\frac{G}{4 !} F \cdot \phi^{4}+\frac{m^{2}}{2} \phi^{2}
$$

где мы используем сокрашенное обозначение $G F \cdot \phi^{4}$ для величины (10). Поскольку присутствие члена (10) явно нарушает масштабную инвариантность, мы провели процедуру “добавить - вычесть” и для массового члена. Отнесем член четвертой степени 
с плюсом к лагранжиану взаимодействия, а такой же член с минусом - к свободному лагранжиану,

$$
\begin{aligned}
L_{0} & =\frac{1}{2} g^{\mu \nu} \frac{\partial \phi}{\partial x^{\mu}} \frac{\partial \phi}{\partial x^{\nu}}-\frac{m^{2}}{2} \phi^{2}-\frac{G}{4 !} F \cdot \phi^{4}-\frac{\epsilon}{4 !} \phi^{4}, \\
L_{\mathrm{int}} & =\frac{g_{0}}{3 !} \phi^{3}+\frac{G}{4 !} F \cdot \phi^{4}+\frac{m^{2}}{2} \phi^{2} .
\end{aligned}
$$

В соответствии с методом квазисредних [2] член четвертой степени в свободном лагранжиане должен быть скомпенсирован так, чтобы новый свободный лагранжиан приводил к нулевым связным четырехчастичным функциям Грина и в результате содержал только квадратичные по полям члены. Проводя вычисления со знаками, отвечаюшими члену в свободном лагранжиане, мы приходим к уравнению компенсации, которое схематически выглядит следуюшим образом: член первого порядка плюс однопетлевые члены плюс двухпетлевые члены, и т.д. При этом в качестве лагранжиана взаимодействия используется член $-G \phi^{4}$. Полученное разложение следует приравнять нулю. Это условие представляет собой уравнение на формфактор $F\left(p_{1}, p_{2}, p_{3}, p_{4}\right)$. Переход к пределу $\epsilon \rightarrow 0$ осушествляется после проведения вычислений, в нашем случае - после получения уравнения компенсации.

Разложение, входящее в это уравнение, явно отличается от разложения по степеням лагранжиана взаимодействия

$$
L_{\mathrm{int}}=\frac{G}{4 !} F \cdot \phi^{4}
$$

знаком константы взаимодействия. В связи с этим подчеркнем, что описанная процедура применима лишь к нарушающим симметрию членам четной степени по полям. Для аналогичных членов нечетной степени, например трилинейных по полям, выполнение уравнения компенсации влечет за собой и обрашение в нуль связной функции Грина, соответствуюшей лагранжиану взаимодействия, поскольку два разложения в этом случае отличаются лишь общим знаком.

Следует отметить, что наличие члена $-G \phi^{4}$ в модифицированном свободном лагранжиане может приводить к возникновению и связных гриновских функций более высокого порядка - шестого, восьмого и т.д. Вообше говоря, следует построить зацепляющуюся систему уравнений компенсации для всех этих гриновских функций. Мы начнем с уравнения для функции Грина четвертого порядка, а вопрос о высших функциях обсудим в дальнейшем.

Получим приближенное уравнение для формфактора четвертого порядка. Прежде всего, ограничимся следующей кинематикой: правые концы имеют нулевые импульсы, а левые концы - импульсы $p$ и $-p$. В разложении ограничимся членами до двухпетлевых включительно. Перечислим их: член первого порядка - точка, три члена второго порядка - простые петли, одна горизонтальная и две вертикальные с перестановкой импульсов у левых концов. В третьем порядке - горизонтальная и две вертикальные двухзвенные цепочки, а также шесть членов: “рюмки” горизонтальные, ножкой вправо и влево, и вертикальные, ножкой вверх и вниз. Число последних удваивается 
благодаря перестановкам левых импульсов $p$ и $-p$. Вообще говоря, в каждой вершине стоит формфактор $F$. Однако мы сможем решить лишь линеаризованное уравнение, которое получаем, оставив в уравнении члены первого и второго порядков, двухзвенную горизонтальную цепочку и рюмку ножкой вправо из членов третьего порядка. Линеаризация проводится следуюшим образом (см. рис. 1): оставляем формфактор $F(p,-p, 0,0) \equiv F\left(p^{2}\right)$ в члене первого порядка и в правых вершинах горизонтальной петли второго порядка, горизонтальной двухзвенной цепочки и горизонтальной рюмки третьего порядка (ножкой вправо). Остальные вершины в этих диаграммах мы рассматриваем как точечные вершины взаимодействия, в которых формфактор заменен его значением в нуле $(F(0)=1)$,

$$
\frac{G}{4 !} F(0) \phi^{4}=\frac{G}{4 !} \phi^{4}
$$
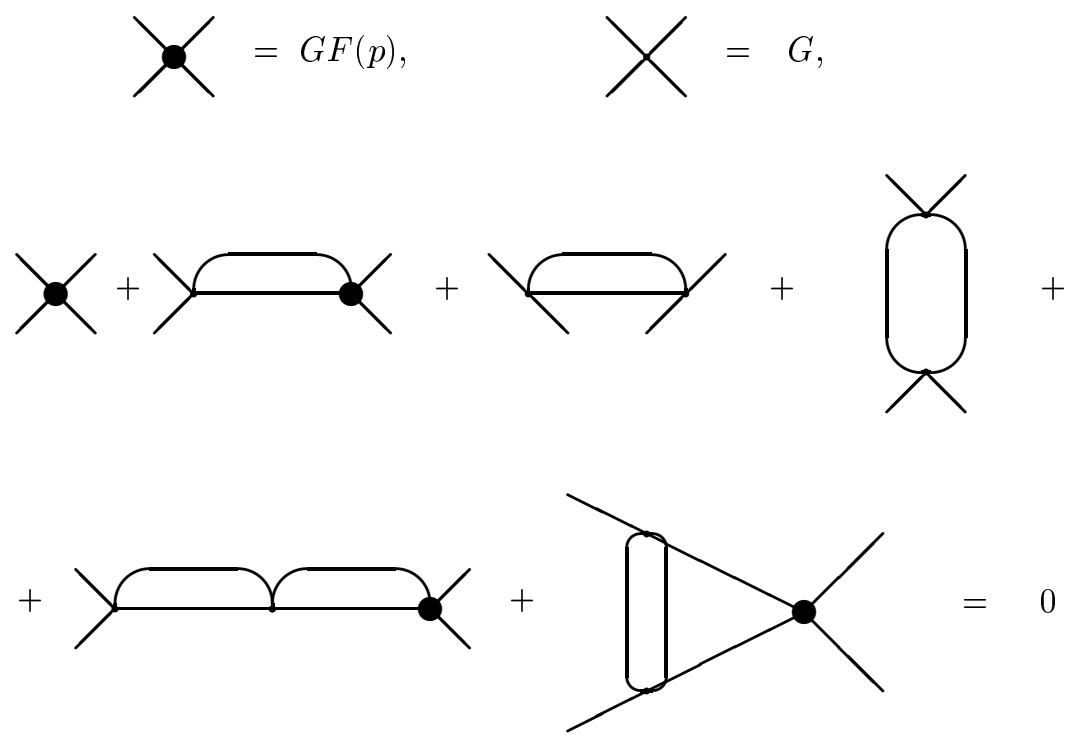

Рис. 1. Графическое представление линеаризованного уравнения компенсации (18).

В вертикальных петлях, которые также будут служить ядром интегрального уравнения, мы подставляем точечные вершины (14). Соответствуюшие интегралы, разумеется, расходятся. Для нахождения убываюшего на бесконечности по импульсам решения для формфактора $F\left(p^{2}\right)$ вводим некоторое обрезание $\Lambda$, сушествование которого будет подтверждено результатом решения уравнения. При этом происходит замена

$$
\int_{0}^{\infty} d q^{2} \rightarrow \Lambda^{2}
$$

Для оценки порядка величины $\Lambda^{2}$ воспользуемся следующим определением:

$$
\Lambda^{2}=\int_{0}^{\infty} F(y) d y
$$


в котором одна из точечных вершин заменена на формфактор. Важным для справедливости подхода является вопрос о сходимости интеграла (15). То же обрезание $\Lambda^{2}$ мы будем подставлять и в логарифмически расходящихся интегралах. Возможное отличие фактического обрезания в этих интегралах от $\Lambda^{2}$ отражается в изменении постоянной $c$, которая войдет в соответствующие выражения. Выяснится, что от значения этой постоянной решение зависеть не будет. Таким образом, формулировка уравнения в рамках сделанных приближений не содержит произвольных предположений.

Уравнение будем рассматривать в евклидовом шестимерном пространстве, к которому переходим с помощью замен (6). В этом случае возникает необходимость знать некоторые угловые интегралы в шестимерном пространстве, которые получаются при вычислении петлевых интегралов в пределе $m^{2} \rightarrow 0$. Вычисляя с помощью известных интегралов (для логарифмического случая см. [9], с. 545), имеем

$$
\begin{aligned}
& \int \frac{d \Omega_{6}}{p^{2}+q^{2}-2 p q \cos \theta}=\frac{4 \pi^{3}}{3}\left(\Theta(x-y)\left(\frac{3}{4 x}-\frac{y}{4 x^{2}}\right)+\Theta(y-x)\left(\frac{3}{4 y}-\frac{x}{4 y^{2}}\right)\right) \\
& \int d \Omega_{6} \ln \left(p^{2}+q^{2}-2 p q \cos \theta\right)=\frac{\pi^{3}}{12}\left(\Theta(x-y)\left(\frac{8 y}{x}-\frac{y^{2}}{x^{2}}+12 \ln x\right)+\right. \\
& \left.\quad+\Theta(y-x)\left(\frac{8 x}{y}-\frac{x^{2}}{y^{2}}+12 \ln y\right)\right) \\
& \int d \Omega_{6}(p q) \ln \left(p^{2}+q^{2}-2 p q \cos \theta\right)=\frac{\pi^{3}}{18}\left(\Theta(x-y)\left(\frac{3 y^{2}}{x}-6 y-\frac{3 y^{3}}{5 x^{2}}\right)+\right. \\
& \left.\quad+\Theta(y-x)\left(\frac{3 x^{2}}{y}-6 x-\frac{3 x^{3}}{5 y^{2}}\right)\right)
\end{aligned}
$$

где $x=p^{2}, y=q^{2}$.

Прежде всего, вычислим однопетлевые члены нулевого и первого порядка разложения по $\mathrm{m}^{2}$. В работе [10] на примере аналогичной задачи показано, что итерационный учет масс приводит к сходящейся процедуре. Имеем для одной такой вертикальной диаграммы $\left(x=p^{2}\right.$, где $p$ - суммарный импульс вдоль петли)

$$
-i \frac{G^{2} \pi^{3}}{2(2 \pi)^{6}}\left(\Lambda^{2}+\frac{1}{3} x \ln \frac{x}{\Lambda^{2}}+2 m^{2} \ln \frac{x}{\Lambda^{2}}-c x\right),
$$

где $\Lambda^{2}$ - квадрат введенного выше обрезания и $c$ - некоторая постоянная, зависяшая от вида обрезающего формфактора. Учтены члены нулевого и первого порядка разложения по $m^{2}$.

Рассмотрим линеаризованное уравнение компенсации (см. рис. 1), соответствуюшее 
сформулированным вьше правилам. Уравнение в этом приближении имеет вид

$$
\begin{aligned}
G F\left(p^{2}\right)= & \frac{G^{2}}{2(4 \pi)^{3}}\left(3 \Lambda^{2}+\frac{2}{3} p^{2} \ln \frac{p^{2}}{\Lambda^{2}}+4 m^{2} \ln \frac{p^{2}}{\Lambda^{2}}-2 c p^{2}\right)- \\
& -\frac{G^{3}}{8(2 \pi)^{9}} \int\left(\frac{1}{3}(p-q)^{2} \ln \frac{(p-q)^{2}}{\Lambda^{2}}+\right. \\
& \left.+2 m^{2} \ln \frac{(p-q)^{2}}{\Lambda^{2}}-c(p-q)^{2}\right) \frac{F\left(q^{2}\right)}{\left(q^{2}+m^{2}\right)^{2}} d^{6} q- \\
& -\frac{3 G^{3} \pi^{3} \Lambda^{2}}{2(2 \pi)^{12}} \int \frac{F\left(q^{2}\right)}{\left(q^{2}+m^{2}\right)^{2}} d^{6} q .
\end{aligned}
$$

Прежде всего, отметим возможность тривиального решения $G=0$. Для поиска нетривиального решения сократим уравнение на $G$. Проводя здесь угловые интегрирования с помощью выражений (16), получаем одномерное интегральное уравнение

$$
\begin{aligned}
F(x)= & \frac{G}{2(4 \pi)^{3}}\left(3 \Lambda^{2}+\frac{2}{3} x \ln \frac{x}{\Lambda^{2}}+4 m^{2} \ln \frac{x}{\Lambda^{2}}-2 c x\right)- \\
& -\frac{3 G^{2} \Lambda^{2}}{4(4 \pi)^{6}} \int_{0}^{\infty} \frac{y^{2} F(y)}{\left(y+m^{2}\right)^{2}} d y-\frac{G^{2}}{18(4 \pi)^{6}}\left(-\frac{1}{20 x^{2}} \int_{0}^{x} \frac{y^{5} F(y)}{\left(y+m^{2}\right)^{2}} d y+\right. \\
& +\frac{3}{4 x} \int_{0}^{x} \frac{y^{4} F(y)}{\left(y+m^{2}\right)^{2}} d y+3 \ln x \int_{0}^{x} \frac{y^{3} F(y)}{\left(y+m^{2}\right)^{2}} d y+3 x \ln x \int_{0}^{x} \frac{y^{2} F(y)}{\left(y+m^{2}\right)^{2}} d y+ \\
& +3 \int_{x}^{\infty} \frac{y^{3} \ln y F(y)}{\left(y+m^{2}\right)^{2}} d y+x \int_{x}^{\infty} \frac{(4+3 \ln y) y^{2} F(y)}{\left(y+m^{2}\right)^{2}} d y+ \\
& \left.+4 \int_{0}^{x} \frac{y^{3} F(y)}{\left(y+m^{2}\right)^{2}} d y+\frac{3 x^{2}}{4} \int_{x}^{\infty} \frac{y F(y)}{\left(y+m^{2}\right)^{2}} d y-\frac{x^{3}}{20} \int_{x}^{\infty} \frac{F(y)}{\left(y+m^{2}\right)^{2}} d y\right)- \\
& -\frac{G^{2} m^{2}}{12(4 \pi)^{6}}\left(-\frac{1}{x^{2}} \int_{0}^{x} \frac{y^{4} F(y)}{\left(y+m^{2}\right)^{2}} d y+\right. \\
& +\frac{8}{x} \int_{0}^{x} \frac{y^{3} F(y)}{\left(y+m^{2}\right)^{2}} d y+12 \ln x \int_{0}^{x} \frac{y^{2} F(y)}{\left(y+m^{2}\right)^{2}} d y+ \\
& \left.+12 \int_{x}^{\infty} \frac{y^{2} \ln y F(y)}{\left(y+m^{2}\right)^{2}} d y+8 x \int_{x}^{\infty} \frac{y F(y)}{\left(y+m^{2}\right)^{2}} d y-x^{2} \int_{x}^{\infty} \frac{F(y)}{\left(y+m^{2}\right)^{2}} d y\right)+ \\
& +\frac{G^{2}}{6(4 \pi)^{6}}\left(\ln \Lambda^{2}+3 c\right)\left(\int_{0}^{\infty} \frac{y^{3} F(y)}{\left(y+m^{2}\right)^{2}} d y+x \int_{0}^{\infty} \frac{y^{2} F(y)}{\left(y+m^{2}\right)^{2}} d y\right)+ \\
& +\frac{G^{2} m^{2}}{(4 \pi)^{6}} \ln \Lambda^{2} \int_{0}^{\infty} \frac{y^{2} F(y)}{\left(y+m^{2}\right)^{2}} d y .
\end{aligned}
$$

Метод нахождения решений подобных уравнений известен [11]. Уравнение (19) последовательным дифференцированием сводится к дифференциальному уравнению

$$
\frac{d^{4}}{d x^{4}}\left(x^{2} \frac{d^{4}}{d x^{4}}\left(x^{2} F(x)\right)\right)=-\beta\left(\frac{F(x)}{\left(x+m^{2}\right)^{2}}+2 m^{2}\left(x \frac{d^{2}}{d x^{2}} \frac{F(x)}{\left(x+m^{2}\right)^{2}}+3 \frac{d}{d x} \frac{F(x)}{\left(x+m^{2}\right)^{2}}\right)\right)
$$


где

$$
\beta=\frac{2 G^{2}}{(4 \pi)^{6}}
$$

Уравнение (20) можно переписать в более удобном виде:

$$
\begin{aligned}
& \left(\left(x \frac{d}{d x}+2\right)\left(x \frac{d}{d x}+1\right)\left(x \frac{d}{d x}\right)\left(x \frac{d}{d x}\right)\left(x \frac{d}{d x}-1\right)\left(x \frac{d}{d x}-1\right) \times\right. \\
& \left.\times\left(x \frac{d}{d x}-2\right)\left(x \frac{d}{d x}-3\right)+\beta x^{2}\right) F(x)=2 \beta m^{2} x\left(F(x)+x \frac{d F}{d x}-x^{2} \frac{d^{2} F}{d x^{2}}\right),
\end{aligned}
$$

где удержаны два члена разложения по $\mathrm{m}^{2}$. Из этой формы уравнения сразу видно, что при $x \rightarrow 0$ имеются восемь независимых асимптотик, коэффициенты при которых мы обозначим следующим образом:

$$
\frac{a_{-2}}{x^{2}}, \quad \frac{a_{-1}}{x}, \quad a_{0}, \quad a_{0 l} \ln x, \quad a_{1} x, \quad a_{1 l} x \ln x, \quad a_{2} x^{2}, \quad a_{3} x^{3}
$$

Восемь независимых асимптотик на бесконечности имеют вид

$$
F_{k}(x) \simeq x^{-3 / 8} \exp \left(4\left(\beta x^{2}\right)^{1 / 8} \exp \left(\frac{i \pi(2 k-1)}{8}\right)\right), \quad k=1,2, \ldots, 8
$$

Четыре асимптотики на бесконечности экспоненциально убывают $(k=3,4,5,6)$, а остальные четыре - возрастают.

Уравнение (21) эквивалентно исходному интегральному уравнению при выполнении определенных граничных условий. Прежде всего, мы можем использовать лишь убываюшие на бесконечности решения. Для получения условий в нуле следует подставить выражение

$$
\frac{\beta}{\left(x+m^{2}\right)^{2}} F(x)=-\frac{d^{4}}{d x^{4}}\left(x^{2} \frac{d^{4}}{d x^{4}}\left(x^{2} F(x)\right)\right)
$$

в интегралы уравнения (19) и провести последовательные интегрирования по частям. Результаты вычислений приведены в приложении (см. формулы (П.1)).

Подставляя выражения (П.1) в уравнение (19), получаем

$$
\begin{aligned}
F(x)= & F(x)-\frac{a_{-2}}{x^{2}}-\frac{a_{-1}}{x}-a_{0 l} \ln x-a_{1 l} x \ln x+\frac{2 G m^{2}}{(4 \pi)^{3}} \ln x+ \\
& +\frac{G \pi^{3}}{2(2 \pi)^{6}}\left(3 \Lambda^{2}\left(1-\frac{G I}{2(4 \pi)^{3}}\right)+\frac{2 x}{3} \ln \frac{x}{\Lambda^{2}}-2 c x\right)+x\left(\ln \Lambda^{2}+3 c\right) a_{1 l}, \\
I= & \int_{0}^{\infty} \frac{y^{2} F(y)}{\left(y+m^{2}\right)^{2}} d y .
\end{aligned}
$$


Отсюда получаем условия (независимо от значений $\Lambda^{2}$ и $c$ )

$$
\begin{gathered}
a_{-2}=0, \quad a_{-1}=0, \quad a_{0 l}=\frac{2 G m^{2}}{(4 \pi)^{3}}, \quad a_{1 l}=\frac{G \pi^{3}}{3(2 \pi)^{6}}=\frac{\sqrt{2 \beta}}{6}, \\
I=\frac{2(4 \pi)^{3}}{G}=\frac{2 \sqrt{2}}{\sqrt{\beta}} .
\end{gathered}
$$

Четыре условия (25) являются граничными условиями к уравнению (21). Комбинация четырех убывающих на бесконечности решений с учетом этих условий дает единственное решение. Его удается явно выразить через известные специальные функции для случая $m^{2}=0$. Действительно, проведем в уравнении (21) с $m^{2}=0$ замену

$$
z=\frac{\beta x^{2}}{2^{8}}
$$

которая сводит уравнение к каноническому виду уравнения Мейера [12] восьмого порядка,

$$
\begin{gathered}
\left(\left(z \frac{d}{d z}+1\right)\left(z \frac{d}{d z}+\frac{1}{2}\right)\left(z \frac{d}{d z}\right)\left(z \frac{d}{d z}\right)\left(z \frac{d}{d z}-\frac{1}{2}\right)\left(z \frac{d}{d z}-\frac{1}{2}\right) \times\right. \\
\left.\times\left(z \frac{d}{d z}-1\right)\left(z \frac{d}{d z}-\frac{3}{2}\right)+z\right) F_{0}(z)=0
\end{gathered}
$$

Условия (25) выделяют решение. Во-первых, четыре убывающих на бесконечности решения всегда можно скомбинировать так, чтобы обратить в нуль три сингулярных асимптотики в нуле, т.е. удовлетворить условиям $a_{-2}=a_{-1}=a_{0 l}=0$. Такое свойство имеет функция Мейера (см. [12])

$$
C G_{08}^{50}(z \mid 3 / 2,1,1 / 2,1 / 2,0,0,-1 / 2,-1) .
$$

Постоянную $C$ мы определяем из коэффициента при $\sqrt{z} \ln z$. Разложение полученной функции Мейера при малых $z$ имеет вид [12]

$$
G_{08}^{50}(z \mid 3 / 2,1,1 / 2,1 / 2,0,0,-1 / 2,-1)=\pi+\frac{16}{3} \sqrt{z} \ln z+\cdots .
$$

Сравнивая коэффициент при $\sqrt{z} \ln z \mathrm{c}(25)$, получаем

$$
C=\frac{\sqrt{2}}{4}
$$

Выгисляя интеграл [13], с. 347, имеем по определению $I(24)$ при $m^{2}=0$ :

$$
I=\int_{0}^{\infty} F(y) d y=\frac{\sqrt{2}}{4} \int_{0}^{\infty} G_{08}^{50}\left(\beta y^{2} / 2^{8} \mid 3 / 2,1,1 / 2,1 / 2,0,0,-1 / 2,-1\right) d y=\frac{2 \sqrt{2}}{\sqrt{\beta}},
$$


что полностью соответствует условию (26).

Итак, решение

$$
F_{0}(x)=\frac{\sqrt{2}}{4} G_{08}^{50}\left(\beta x^{2} / 2^{8} \mid 3 / 2,1,1 / 2,1 / 2,0,0,-1 / 2,-1\right)
$$

удовлетворяет всем условиям (25), а следовательно, и исходному уравнению (19) при $m^{2}=0$, которое является (приближенным) уравнением компенсации. Это решение является нетривиальным, содержашим размерный параметр $G$, следовательно, оно приводит к нарушению исходной масштабной симметрии. Разумеется, всегда есть и возможность тривиального решения $G F(x)=0$. Заметим, что отмеченные условия удовлетворяются независимо от значения формфактора в нуле. Равенство $F(0)=1$ будет дополнительным условием в дальнейших рассуждениях.

Проведем итерационный учет $m^{2}$. Будем искать поправку к решению уравнения $(21)$ в виде

$$
F(x)=F_{0}(x)+\Delta F(x) .
$$

Подставляя (32) в (21), получаем уравнение в первом порядке по $\mathrm{m}^{2}$,

$$
\begin{gathered}
\left(\left(x \frac{d}{d x}+2\right)\left(x \frac{d}{d x}+1\right)\left(x \frac{d}{d x}\right)\left(x \frac{d}{d x}\right)\left(x \frac{d}{d x}-1\right)\left(x \frac{d}{d x}-1\right)\left(x \frac{d}{d x}-2\right) \times\right. \\
\left.\times\left(x \frac{d}{d x}-3\right)+\beta x^{2}\right) \Delta F(x)=2 \beta m^{2} x\left(F_{0}(x)+x \frac{d F_{0}}{d x}-x^{2} \frac{d^{2} F_{0}}{d x^{2}}\right) .
\end{gathered}
$$

Из уравнения (33) можно точно определить несколько членов разложения $\Delta F(x)$ при малых $x$. Действительно, рассмотрим выражение

$$
\begin{aligned}
\bar{\Delta} F(x) & =\frac{2 m^{2}}{x}\left(F_{0}(x)+x \frac{d F_{0}}{d x}-x^{2} \frac{d^{2} F_{0}}{d x^{2}}\right)= \\
& =2 m^{2}\left(\frac{\pi}{2 \sqrt{2} x}+\frac{2 G}{3(4 \pi)^{3}}\left(\ln (\sqrt{\beta} x)+4 \gamma-\frac{23}{6}\right)+\frac{\pi \sqrt{2} G^{2}}{96(4 \pi)^{6}} x \ln x+O(x)\right)
\end{aligned}
$$

где $\gamma=0.577215665 \ldots$ - постоянная Эйлера. Подставляя выражение (34) в уравнение (33), мы убеждаемся, что оно является решением неоднородного дифференциального уравнения вплоть до членов порядка $x^{3}$, поскольку дифференциальный оператор слева обращает в нуль и выписанные явно члены, и последующие члены вплоть до указанного порядка. Нас прежде всего интересуют именно члены, выписанные в (34), так как они имеют прямое отношение к граничным условиям. В самом деле, в выражении (34) члены, пропорциональные $x^{-1}, \ln x, x \ln x$, нарушают граничные условия. Следовательно, 
мы должны добавить к выражению (34) комбинацию решений однородного уравнения, с тем чтобы добиться выполнения граничных условий. Получаем окончательно

$$
\begin{aligned}
\Delta F(x)= & \bar{\Delta} F(x)-\frac{\pi^{2} Y}{8} G_{08}^{50}\left(\beta x^{2} / 2^{8} \mid 3 / 2,1,1 / 2,1 / 2,-1 / 2,0,0,-1\right)- \\
& -\frac{2 Y}{3} G_{08}^{50}\left(\beta x^{2} / 2^{8} \mid 3 / 2,1,1 / 2,0,0,1 / 2,-1 / 2,-1\right)- \\
& -\pi Y\left(\gamma+\ln 2-\frac{43}{48}\right) G_{08}^{50}\left(\beta x^{2} / 2^{8} \mid 3 / 2,1,1 / 2,1 / 2,0,0,-1 / 2,-1\right),
\end{aligned}
$$

где $Y=G m^{2} /\left(2(4 \pi)^{3}\right)$. С помощью этого выражения можно точно найти $F(0)$. При этом надо иметь в виду, что наличие члена, пропорционального $\ln x$ при $x \rightarrow 0$, является следствием разложения по $m^{2}$ при $x \gg m^{2}$. Возврашаясь к соответствуюшим вычислениям, убеждаемся, что при $x \rightarrow 0$ следует заменить $\ln x$ на $\ln \left(4 m^{2}\right)$, тогда

$$
F(0)=\frac{\pi \sqrt{2}}{4}+Y\left(4 \ln Y+\left(16-\pi^{2}\right) \gamma+\left(14-\pi^{2}\right) \ln 2-\frac{122}{9}+\pi^{2} \frac{42}{48}\right)
$$

При $Y=0$ получаем $F(0)=1.11072$. Условие $F(0)=1$ определяет значение параметра $Y$, связанного с массой (см. (35)),

$$
Y=0.005789
$$

Таким образом, найденное нами решение со значением $Y$ (37) удовлетворяет всем необходимым условиям. Подчеркнем, что (37) представляет собой условие на массу скалярного поля. Отметим, что малое значение $Y(37)$ вполне оправдывает учет лишь первого члена разложения по $m^{2}$. Второе решение уравнения $F(0)=1$, имеющее порядок единицы, мы отбрасываем, так как оно не согласуется с разложением решения по $\mathrm{m}^{2}$.

Мы уже отмечали, что, вообще говоря, следует рассматривать всю цепочку зацепляющихся уравнений компенсации, в том числе и для связных функций Грина с шестью, восемью и т.д. концами. Соответствующие уравнения будут содержать неоднородные части, выраженные через функции Грина более низкого порядка, и однородные части, пропорциональные соответствующим формфакторам, например, с шестью концами $F_{6}$. Если принять обрашение в нуль связной четверной функции Грина, то неоднородная часть уравнения компенсации для $F_{6}$ обращается в нуль и, следовательно, всегда сушествует тривиальное решение $F_{6}=0$. Точно такие же рассуждения приводят к выводу о возможности тривиального решения и для всех последующих вершин. Можно, конечно, исследовать и возможности сушествования нетривиальных решений. Однако цель настоящей работы состоит в том, чтобы показать наличие хотя бы одного нетривиального решения. Поэтому мы ограничимся лишь случаем нетривиального решения для связной функции Грина четвертого порядка при тривиальных решениях для всех высших функций Грина. Вопрос об уравнении компенсации для функции Грина второго порядка, которая определяет массу скалярного поля, будет рассмотрен особо. 
Переход от линеаризованного уравнения (18) к нелинейному с учетом всех необходимых диаграмм приводит к задаче, аналитическое исследование которой невозможно. Оценка влияния нелинейности на значение формфактора в нуле будет проведена ниже. Возможно, в дальнейшем будут применены численные методы решения таких уравнений. Представляется, что опыт, приобретенный при получении нетривиального решения, поможет в формулировке численных задач. Можно предположить, что большое значение при этом будет иметь результат (37), согласно которому решение может сушествовать только при определенном соотношении между размерной константой связи и массой.

\section{4. УРАВНЕНИЕ БЕТЕ-СОЛПИТЕРА И НУЛЕВОЕ ВОЗБУЖДЕНИЕ}

Как известно, нарушение симметрии обязано сопровождаться появлением в спектре возбуждения с нулевой массой [1], [2], [14]. Рассмотрим эту проблему в том же приближении, что и вьше. При формулировке уравнения на связанное состояние нужно иметь в виду, что здесь действует истинное взаимодействие (13), которое было отнесено в лагранжиан взаимодействия и осталось, естественно, нескомпенсированным. Уравнение Бете-Солпитера для безмассового связанного состояния двух скалярных полей в этом случае имеет вид

$$
\begin{aligned}
\Psi(x)= & \frac{G \pi^{3} \Lambda^{\prime}}{2(2 \pi)^{6}}-\frac{G^{2} \pi^{6} \Lambda^{2} \Lambda^{\prime}}{2(2 \pi)^{12}}+\frac{G^{2} \pi^{6}}{18(2 \pi)^{12}}\left(-\frac{1}{20 x^{2}} \int_{0}^{x} \frac{y^{5}}{\left(y+m^{2}\right)^{2}} \Psi(y) d y+\right. \\
& +\frac{3}{4 x} \int_{0}^{x} \frac{y^{4}}{\left(y+m^{2}\right)^{2}} \Psi(y) d y+3 \ln x \int_{0}^{x} \frac{y^{3}}{\left(y+m^{2}\right)^{2}} \Psi(y) d y+ \\
& +4 \int_{0}^{x} \frac{y^{3}}{\left(y+m^{2}\right)^{2}} \Psi(y) d y+3 x \ln x \int_{0}^{x} \frac{y^{2}}{\left(y+m^{2}\right)^{2}} \Psi(y) d y+ \\
& +3 \int_{x}^{\infty} \frac{y^{3} \ln y}{\left(y+m^{2}\right)^{2}} \Psi(y) d y+x \int_{x}^{\infty} \frac{y^{2}(4+3 \ln y)}{\left(y+m^{2}\right)^{2}} \Psi(y) d y+ \\
& \left.+\frac{3 x^{2}}{4} \int_{x}^{\infty} \frac{y}{\left(y+m^{2}\right)^{2}} \Psi(y) d y-\frac{x^{3}}{20} \int_{x}^{\infty} \frac{\Psi(y)}{y^{2}} d y\right)-\frac{G^{2} \pi^{6}}{6(2 \pi)^{12}} \times \\
& \times\left(\ln \Lambda^{2}+3 c\right)\left(\int_{0}^{\infty} \frac{y^{3}}{\left(y+m^{2}\right)^{2}} \Psi(y) d y+x \int_{0}^{\infty} \frac{y^{2}}{\left(y+m^{2}\right)^{2}} \Psi(y) d y\right),
\end{aligned}
$$

где

$$
\Lambda^{\prime}=\int_{0}^{\infty} \frac{y^{2}}{\left(y+m^{2}\right)^{2}} \Psi(y) d y .
$$

Сравнивая уравнение (38) с уравнением компенсации (19), мы видим основное отличие в знаке перед ядром интегрального уравнения. Еще раз напомним, что уравнение компенсации есть условие обрашения в нуль полной суммы разложения по $G$ в модифицированном свободном лагранжиане в выражении (11), и потому члены первого и третьего порядков находятся в одной, скажем, левой части уравнения, в то время как в уравнении Бете-Солпитера соответствуюшие члены находятся в разных частях уравнения. 
Знак перед ядром очень важен. Он означает, что и в дифференциальном уравнении знак перед членом, пропорциональным $\beta$, также меняется,

$$
\begin{gathered}
\left(\left(x \frac{d}{d x}+2\right)\left(x \frac{d}{d x}+1\right)\left(x \frac{d}{d x}\right)\left(x \frac{d}{d x}\right)\left(x \frac{d}{d x}-1\right)\left(x \frac{d}{d x}-1\right) \times\right. \\
\left.\times\left(x \frac{d}{d x}-2\right)\left(x \frac{d}{d x}-3\right)-\beta \frac{x^{4}}{\left(x+m^{2}\right)^{2}}\right) \Psi(x)=0 .
\end{gathered}
$$

Легко видеть, что в силу отсутствия в неоднородной части члена, пропорционального $x \ln x$, граничные условия имеют вид

$$
a_{-2}=a_{-1}=a_{0 l}=a_{1 l}=0 .
$$

Изменение знака перед $\beta$ приводит к тому, что асимптотики на бесконечности меняются,

$$
\Psi_{k}(x) \simeq x^{-3 / 8} \exp \left(4\left(\beta x^{2}\right)^{1 / 8} \exp \left(\frac{i \pi k}{4}\right)\right), \quad k=1,2, \ldots, 8
$$

Теперь мы имеем три экспоненциально убывающих решения $(k=3,4,5)$, два осциллируюших решения со степенным убыванием $(k=2,6)$ и три возрастающих. Используя первые пять решений, которые допускают определение интегралов на бесконечности, мы удовлетворяем четырем граничным условиям в нуле (40). Из граничных условий (см. приложение) следует также, что $\Lambda^{\prime}=0$, так что неоднородная часть уравнения (38) обрашается в нуль. В результате приходим к выводу, что уравнение (38) на связанное состояние имеет решение, которое для случая $m^{2}=0$ выписывается явно:

$$
\Psi(x)=A G_{08}^{40}\left(\beta x^{2} / 2^{8} \mid 3 / 2,1,1 / 2,0,1 / 2,0,-1 / 2,-1\right)
$$

где постоянная $A$ определяется из условия нормировки волновой функции Бете-Солпитера.

Полученное решение доказывает сушествование нулевого возбуждения [1], [2], [14] в рассматриваемом случае. Разумеется, само определение уравнения Бете-Солпитера возможно лишь в случае существования нетривиального решения уравнения компенсации и, следовательно, наличия взаимодействия (13). Обязательная связь сушествования решения уравнения компенсации с наличием нулевого возбуждения находится в полном соответствии с подходом квазисредних Боголюбова [2].

Интересно отметить, что при учете трилинейного взаимодействия $g \phi^{3}$ в ядре уравнения (38) масса связанного состояния становится отличной от нуля, что вполне понятно, поскольку само взаимодействие $g \phi^{3}$ приводит к наличию размерного параметра $\Lambda_{3}$ и тем самым нарушает масштабную инвариантность. 


\section{5. УРАВНЕНИЕ КОМПЕНСАЦИИ ДЛЯ МАССЫ СКАЛЯРНОГО ПОЛЯ}

Обратимся к лагранжиану взаимодействия (12). В нем "инородным телом" является массовый член. Для решения этой проблемы нужно построить уравнение компенсации для функции Грина второго порядка. Рассмотрим это уравнение с учетом решения (32) и трилинейного взаимодействия.Уравнение компенсации означает обрашение в нуль суммарного вклада взаимодействия (12) в массу. В первом приближении вклад четверного взаимодействия дается диаграммой первого порядка “пузырь", а вклад трилинейного - однопетлевой диаграммой (см. рис. 2).

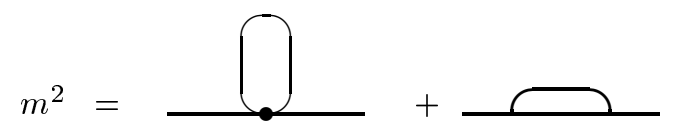

Рис. 2. Уравнение компенсации для массы скалярного поля.

Положив импульсы внешних концов равными нулю, для четверной вершины имеем как раз решение (32). В результате получаем уравнение компенсации для массы

$$
\begin{aligned}
m^{2}= & -\frac{G}{(2 \pi)^{6}} \int \frac{F\left(q^{2}\right) d^{6} q}{q^{2}+m^{2}}-\frac{g^{2}}{(2 \pi)^{6}} \int \frac{d^{6} q}{\left(q^{2}+m^{2}\right)^{2}}= \\
= & -\frac{G}{2(4 \pi)^{3}} \int_{0}^{\infty} y d y\left(F_{0}(y)+\Delta F(y)\right)+\frac{G m^{2}}{2(4 \pi)^{3}} \int_{0}^{\infty} d y F_{0}(y)- \\
& -\frac{g^{2}}{2(4 \pi)^{3}} \int_{0}^{\infty} \frac{y^{2} d y}{\left(y+m^{2}\right)^{2}} .
\end{aligned}
$$

Здесь в диаграмме “пузырь" мы провели разложение по $m^{2}$ и учли нулевой и первый порядки разложения. Непосредственным вычислением с использованием выражений (31), (34), (35) получаем, что член нулевого порядка обрашается в нуль, а вклад первого порядка равен $3 m^{2}$. Петля, которая описывается последним членом в (43), дает квадратичную расходимость. Мы считаем, что в исходной теории (5) вводится некоторое обрезание $\Lambda_{3}$, отражающее физическое ограничение области применимости этой теории. В результате приходим к следуюшему уравнению компенсации для массы в предположении $m \ll \Lambda_{3}$

$$
m^{2}=3 m^{2}-\frac{g^{2}}{2(4 \pi)^{3}} \Lambda_{3}^{2} .
$$

Подчеркнем, что в случае тривиального решения $G=0$ первый член в правой части уравнения (44) отсутствует и мы имеем отрицательный квадрат массы, т.е. тахионное решение. Для нетривиального решения

$$
m^{2}=\frac{g^{2}}{4(4 \pi)^{3}} \Lambda_{3}^{2}
$$


Как известно, скалярный тахион приводит к неустойчивости при малых полях. Поэтому восстановление нормального знака квадрата массы, которое достигается при осушествлении нетривиального решения, соответствует переходу к более устойчивому состоянию.

Итак, значение массы скаляра определяется через исходные параметры теории $g$ и $\Lambda_{3}$. Значение параметра $Y(37)$ дает связь массы скалярного поля и константы эффективного четверного взаимодействия $G$. Таким образом, все параметры, входяшие в нетривиальное решение, определены через исходные параметры.

Подчеркнем, что под $\Lambda_{3}$ здесь мы понимаем некоторую граничную энергию, дающую реальное обрезание соответствующих интегралов. В реальном четырехмерном случае это может быть, например, планковская энергия $1.22 \cdot 10^{19}$ ГэВ. Отметим также, что в реалистических моделях без элементарных скаляров (см., например, [6], [7]) квадратичные расходимости в массах исходных полей отсутствуют. Следует ожидать, что соотношения, аналогичные (44), также приведут к связям между параметрами теории и упомянутой граничной энергией, входящей в логарифмически расходящиеся члены.

Окончательный вид для эффективного лагранжиана теории после нарушения масштабной инвариантности таков:

$$
L=\frac{1}{2} \frac{\partial \phi}{\partial x^{\mu}} \frac{\partial \phi}{\partial x^{\mu}}-\frac{m^{2}}{2} \phi^{2}+\frac{g}{3 !} \phi^{3}+\frac{G}{4 !} F \cdot \phi^{4},
$$

где формфактор $F$ является решением уравнения компенсации.

\section{6. ОЦЕНКА ВЛИЯНИЯ НЕЛИНЕЙНОСТИ}

До сих пор наши выводы основывались на линеаризованном приближении. Результат - убываюший формфактор - указывает на область применимости приближения. Оно заведомо неприменимо при больших импульсных переменных, поскольку эффективная константа связи становится малой по сравнению с константой $G$, которая использовалась для определения ядра интегрального уравнения. Мы можем провести грубый учет влияния нелинейности, используя следующую процедуру. Пусть при малых $x$ справедливо линейное уравнение $(20) \mathrm{c}^{2}=0$,

$$
\frac{d^{4}}{d x^{4}}\left(x^{2} \frac{d^{4}}{d x^{4}}\left(x^{2} F(x)\right)\right)=-\beta \frac{F(x)}{x^{2}}, \quad \beta=\frac{2 G^{2}}{(4 \pi)^{6}}
$$

Будем считать это уравнение справедливым при $x \leqslant x_{0}$ вместе с соответствуюшими граничными условиями (25), в то время как при $x \geqslant x_{0}$ нужно учитьвать нелинейность. Обратим внимание на то, что коэффициент $\beta$ пропорционален $G^{2}$, т.е. содержит квадрат формфактора. Поэтому при $x \geqslant x_{0}$ мы вместо (47) используем уравнение

$$
\frac{d^{4}}{d x^{4}}\left(x^{2} \frac{d^{4}}{d x^{4}}\left(x^{2} F(x)\right)\right)=-\beta \frac{F^{3}(x)}{x^{2}} .
$$


В таком приближении правильно передается поведение правых частей уравнений при мальх (47) и при очень больших (48) аргументах. В промежуточной области имеется разрыв правой части при $x=x_{0}$. Это означает разрыв восьмой производной искомого решения. Как будет видно ниже, достаточно потребовать неперерывности функции и ее производных до пятого порядка. Введем переменную $y=\sqrt{\beta} x$. Нетрудно видеть, что при $y \rightarrow \infty$ уравнение (48) определяет следующую убывающую асимптотику:

$$
F(y) \simeq \frac{b}{y^{2}}-\frac{6 b^{3}}{5 ! 7 ! y^{4}}+\frac{12 b^{5}}{7 ! 7 ! 8 ! y^{6}}+\cdots,
$$

где $b$ - некоторая постоянная. В то же время уравнение (47) при учете граничных условий имеет следуюшее решение в области $\left(0, y_{0}\right)$ :

$$
\begin{aligned}
F(y)= & \frac{\sqrt{2}}{4} G_{08}^{50}\left(y^{2} / 256 \mid 3 / 2,1,1 / 2,1 / 2,0,0,-1 / 2,-1\right)+ \\
& +C_{1} G_{08}^{30}\left(y^{2} / 256 \mid 3 / 2,1,1 / 2,1 / 2,0,0,-1 / 2,-1\right)+ \\
& +C_{2} G_{08}^{30}\left(y^{2} / 256 \mid 3 / 2,1,0,1 / 2,1 / 2,0,-1 / 2,-1\right)+ \\
& +C_{3} G_{08}^{10}\left(y^{2} / 256 \mid 3 / 2,1,1 / 2,1 / 2,0,0,-1 / 2,-1\right)+ \\
& +C_{4} G_{08}^{10}\left(y^{2} / 256 \mid 1,3 / 2,1 / 2,1 / 2,0,0,-1 / 2,-1\right)
\end{aligned}
$$

где $C_{i}, i=1,2,3,4,-$ некоторые постоянные. Появление дополнительных членов с этими постоянными, помноженными на растущие на бесконечности функции Мейера, связано с тем, что теперь убывание на бесконечности обеспечивается асимптотикой (49) и, следовательно, в области $\left(0, y_{0}\right)$ мы обязаны использовать все решения уравнения (47), удовлетворяющие граничным условиям в нуле. Первый член в (50) - это решение (31), которое получено ранее. Начнем последовательньй учет новых членов с нулевого приближения, в котором в области $\left(0, y_{0}\right)$ имеем это старое решение, т.е. все $C_{i}=0$. Это решение будем сшивать с решением (49) в точке $y_{0}$. Мы убедимся, что в (49) достаточно учитывать один первый член. Тогда из непрерывности функции и ее производной получаем следующую систему уравнений:

$$
\begin{aligned}
& \frac{\sqrt{2}}{4} G_{08}^{50}\left(y_{0}^{2} / 256 \mid 3 / 2,1,1 / 2,1 / 2,0,0,-1 / 2,-1\right)-\frac{b}{y_{0}^{2}}=0, \\
& \frac{\sqrt{2}}{4} G_{08}^{50}\left(y_{0}^{2} / 256 \mid 3 / 2,1,1,1 / 2,1 / 2,0,-1 / 2,-1\right)-\frac{b}{y_{0}^{2}}=0 .
\end{aligned}
$$

Решение этой системы

$$
y_{0}=8.4980, \quad b=7.5055 \text {. }
$$

При этом второй член в асимптотике $(49)$ в точке $y_{0}$ составляет $7.7 \cdot 10^{-6}$ от первого, что оправдывает учет лиш первого члена. Значение формфактора в нуле не изменяется, $F(0)=1.1107$. Учтем теперь два дополнительных члена в (50) с коэффициентами $C_{1}$ и $C_{2}$, которые при малых $y$ дают бо́льший вклад, чем оставшиеся два члена. В этом 
случае нужно сшить значения функции и ее производных до третьей производной включительно. Соответствующая система четырех уравнений получается с помощью правил дифференцирования $G$-функций Мейера [12]. Решение ее имеет вид

$$
y_{0}=17.635, \quad b=9.410, \quad C_{1}=0.0166, \quad C_{2}=-0.0538 \text {. }
$$

Значение формфактора в нуле

$$
F(0)=\frac{\pi \sqrt{2}}{4}+\frac{C_{2}}{\pi}=1.0936 .
$$

Учтем оставшиеся члены с коэффициентами $C_{3}, C_{4}$. Будем считать их, а также отклонения от решения (53), которые мы обозначили через $\Delta y_{0}$ и т.д., малыми. Тогда, сшивая производные до пятого порядка включительно, получаем систему из шести линейных уравнений, решение которой имеет вид

$$
\begin{array}{lll}
\Delta y_{0}=1.457, & \Delta b=1.032, & \Delta C_{1}=-0.0094, \\
\Delta C_{2}=0.0223, & C_{3}=-0.0249, & C_{4}=0.0136 .
\end{array}
$$

Подставляя в (54) вместо $C_{2}$ сумму $C_{2}+\Delta C_{2}$ из $(53)$ и $(55)$, получаем

$$
F(0)=1.1007 \text {. }
$$

Последовательность чисел $1.1107,1.0936,1.1007$ для значения $F(0)$ указьвает на устойчивость результата относительно учета поправок на нелинейность.

\section{7. ЗАКЛЮЧЕНИЕ}

На основании полученных результатов мы высказываем гипотезу о том, что в рассматриваемой модели действительно сушествует нетривиальное решение, нарушаюшее исходную масштабную инвариантность и приводящее к спонтанному возникновению эффективного взаимодействия в лагранжиане (46), которое действует в области импульсного пространства, ограниченной в соответствии со значением параметра $G$. Эффективный формфактор $F(p)$ в соответствии с полученными результатами убывает при $p^{2} \rightarrow$ $\pm \infty$, т.е. и для пространственноподобных, и для времениподобных импульсов. Подтверждено наличие возбуждения с нулевой массой, которое обязано сушествовать при спонтанном нарушении инвариантности.

Мы исходили из асимптотически свободной теории скалярного поля (в шестимерном пространстве), а в результате получили вполне определенную теорию с взаимодействием, нарушаюшим масштабную инвариантность. Возникающие при этом размерные параметры $G^{-1 / 2}$ и $m$ пропорциональны параметру $\Lambda_{3}$, определяющему исходное асимптотически свободное взаимодействие. Необходимо еще раз подчеркнуть, что полученное новое взаимодействие является эффективным, что, прежде всего, отражается в сушествовании формфактора $F(p)$, который, собственно, и является решением уравнения 
компенсации метода квазисредних Боголюбова. На бесконечности по импульсам теория вновь становится асимптотически свободной.

Важно, что рассмотренная задача имеет согласованное решение только при включении тройного взаимодействия $g \phi^{3}$. Действительно, хотя уравнение компенсации (18) не содержит вкладов этого взаимодействия, но отличная от нуля масса скалярного поля получается только при $g \neq 0$. В противном случае значение формфактора $F(p)$ в нуле оказывается не равным единице. Вообще говоря, заранее нельзя исключить возможность того, что условие $F(0)=1$ реализуется в случе $m=0$. Однако опыт рассмотрения задачи, в частности в настоящей работе, показывает, что выполнение этого условия требует какого-то особого стечения обстоятельств, которое вряд ли реализуемо в разумных теориях. На самом деле рассматриваемая задача определяется не одним уравнением компенсации (18), а системой уравнений (18), (43), в которой явно присутствует вклад тройного взаимодействия.

Следует подчеркнуть, что возможность существования нетривиального решения кардинальным образом зависит от выбора теории. Можно проиллюстрировать это положение на примере сравнения различных сигнатур шестимерного пространства. А именно, если вместо сигнатуры $1+5$ выбрать сигнатуру $3+3$, то в определении (6) перехода к евклидовым координатам изменится знак перед $i d^{6} p$. В результате изменятся знаки у однопетлевых интегралов. Для взаимодействия четвертой степени мы восстанавливаем все приведенные результаты простой заменой $G \rightarrow-G$. Однако однопетлевой интеграл с двумя трилинейными вершинами неизбежно меняет знак, и соотношение (44) приводит к тахионной массе. В результате мы заключаем, что для сигнатуры $3+3$ устойчивым является только тривиальное решение $G=0$.

Разумеется, мы основываем свои выводы лишь на точном решении приближенного уравнения. Однако возможные качественные свойства решений, проявившиеся в рассмотренной модельной задаче, нужно будет иметь в виду при изучении проблем спонтанного нарушения симметрии и в более реалистических случаях, когда никакой надежды на аналитические решения соответствующих уравнений нет, а применимыми будут лишь объемные численные методы. К интересным качественным результатам можно отнести наличие связей между параметрами задачи и естественное возникновение малого параметра $Y(37)$. Существен также результат об устойчивости нетривиального решения. То, что модельный учет нелинейности не приводит к кардинальным изменениям свойств решений, является дополнительным аргументом в пользу предлагаемого подхода.

Результирующая теория является нелокальной, и может возникнуть вопрос о вьполнении в ней основных принципов - причинности и унитарности. Исходная теория (5) полностью соответствует этим принципам. Следует ожидать, что ее решения, в том числе и нетривиальные, должны также удовлетворять этим условиям. Поэтому рассмотренньй пример можно также рассматривать и как продвижение на пути построения непротиворечивой нелокальной теории. При этом, основьваясь на полученных результатах, мы вправе предположить, что непротиворечивой такая теория может быть лишш для та-

2 Теоретическая и математическая физика, т. 140, № 3, 2004 г. 
кого формфактора, который реализует нетривиальное решение для исходно локальной теории.

Вне всякого сомнения, возможность спонтанного возникновения эффективного взаимодействия, содержашего размерный параметр, представляет большой интерес для изучения проблем, лежащих за пределами стандартной теории. В частности, явление спонтанного возникновения эффективного взаимодействия в случае его осушествления в реальных физических теориях, например в электрослабой теории, могло бы значительно продвинуть наше понимание основных принципов. Некоторые соображения по этому поводу изложены в работах [6], [7]. Дальнейшие результаты в этом направлении и их связь с возможными отклонениями наблюдаемых эффектов от предсказаний стандартной теории будут представлены в последуюших публикациях.

\section{ПРИЛОЖКНИЕ}

Ниже приведены формулы интегрирования по частям выражений, входяших в уравнение (19):

$$
\begin{aligned}
& \beta \int_{0}^{x} \frac{y^{2} F(y)}{\left(y+m^{2}\right)^{2}} d y=-x^{2} \frac{d^{3}}{d x^{3}}\left(x^{2} \frac{d^{4}}{d x^{4}}\left(x^{2} F(x)\right)\right)+ \\
& +2 x \frac{d^{2}}{d x^{2}}\left(x^{2} \frac{d^{4}}{d x^{4}}\left(x^{2} F(x)\right)\right)-2 \frac{d}{d x}\left(x^{2} \frac{d^{4}}{d x^{4}}\left(x^{2} F(x)\right)\right)+12 a_{1 l}, \\
& \beta \int_{0}^{x} \frac{y^{3} F(y)}{\left(y+m^{2}\right)^{2}} d y=-x^{3} \frac{d^{3}}{d x^{3}}\left(x^{2} \frac{d^{4}}{d x^{4}}\left(x^{2} F(x)\right)\right)+3 x^{2} \frac{d^{2}}{d x^{2}}\left(x^{2} \frac{d^{4}}{d x^{4}}\left(x^{2} F(x)\right)\right)- \\
& -6 x \frac{d}{d x}\left(x^{2} \frac{d^{4}}{d x^{4}}\left(x^{2} F(x)\right)\right)+6 x^{2} \frac{d^{4}}{d x^{4}}\left(x^{2} F(x)\right)-12 a_{0 l}, \\
& \beta \int_{0}^{x} \frac{y^{4} F(y)}{\left(y+m^{2}\right)^{2}} d y=-x^{4} \frac{d^{3}}{d x^{3}}\left(x^{2} \frac{d^{4}}{d x^{4}}\left(x^{2} F(x)\right)\right)+4 x^{3} \frac{d^{2}}{d x^{2}}\left(x^{2} \frac{d^{4}}{d x^{4}}\left(x^{2} F(x)\right)\right)- \\
& -12 x^{2} \frac{d}{d x}\left(x^{2} \frac{d^{4}}{d x^{4}}\left(x^{2} F(x)\right)\right)+24 x^{3} \frac{d^{4}}{d x^{4}}\left(x^{2} F(x)\right)-24 x^{2} \frac{d^{3}}{d x^{3}}\left(x^{2} F(x)\right)+ \\
& +48 x \frac{d^{2}}{d x^{2}}\left(x^{2} F(x)\right)-48 \frac{d}{d x}\left(x^{2} F(x)\right)+48 a_{-1}, \\
& \beta \int_{0}^{x} \frac{y^{5} F(y)}{\left(y+m^{2}\right)^{2}} d y=-x^{5} \frac{d^{3}}{d x^{3}}\left(x^{2} \frac{d^{4}}{d x^{4}}\left(x^{2} F(x)\right)\right)+5 x^{4} \frac{d^{2}}{d x^{2}}\left(x^{2} \frac{d^{4}}{d x^{4}}\left(x^{2} F(x)\right)\right)- \\
& -20 x^{3} \frac{d}{d x}\left(x^{2} \frac{d^{4}}{d x^{4}}\left(x^{2} F(x)\right)\right)+60 x^{4} \frac{d^{4}}{d x^{4}}\left(x^{2} F(x)\right)-120 x^{3} \frac{d^{3}}{d x^{3}}\left(x^{2} F(x)\right)+ \\
& +360 x^{2} \frac{d^{2}}{d x^{2}}\left(x^{2} F(x)\right)-720 x \frac{d}{d x}\left(x^{2} F(x)\right)+720 x^{2} F(x)-720 a_{-2}, \\
& \beta \int_{x}^{\infty} \frac{F(y)}{\left(y+m^{2}\right)^{2}} d y=\frac{d^{3}}{d x^{3}}\left(x^{2} \frac{d^{4}}{d x^{4}}\left(x^{2} F(x)\right)\right), \\
& \beta \int_{x}^{\infty} \frac{y F(y)}{\left(y+m^{2}\right)^{2}}=x \frac{d^{3}}{d x^{3}}\left(x^{2} \frac{d^{4}}{d x^{4}}\left(x^{2} F(x)\right)\right)-\frac{d^{2}}{d x^{2}}\left(x^{2} \frac{d^{4}}{d x^{4}}\left(x^{2} F(x)\right)\right),
\end{aligned}
$$




$$
\begin{aligned}
& \beta \int_{x}^{\infty} \frac{y^{2} \ln y F(y)}{\left(y+m^{2}\right)^{2}} d y=x^{2} \ln x \frac{d^{3}}{d x^{3}}\left(x^{2} \frac{d^{4}}{d x^{4}}\left(x^{2} F(x)\right)\right)-(2 x \ln x+x) \times \\
& \times \frac{d^{2}}{d x^{2}}\left(x^{2} \frac{d^{4}}{d x^{4}}\left(x^{2} F(x)\right)\right)+(2 \ln x+3) \frac{d}{d x}\left(x^{2} \frac{d^{4}}{d x^{4}}\left(x^{2} F(x)\right)\right)- \\
&-2 x \frac{d^{4}}{d x^{4}}\left(x^{2} F(x)\right)+2 \frac{d^{3}}{d x^{3}}\left(x^{2} F(x)\right), \\
& \beta \int_{x}^{\infty} \quad \frac{y^{2} F(y)}{\left(y+m^{2}\right)^{2}} d y=x^{2} \frac{d^{3}}{d x^{3}}\left(x^{2} \frac{d^{4}}{d x^{4}}\left(x^{2} F(x)\right)\right)-2 x \frac{d^{2}}{d x^{2}}\left(x^{2} \frac{d^{4}}{d x^{4}}\left(x^{2} F(x)\right)\right)+ \\
& \quad+2 \frac{d}{d x}\left(x^{2} \frac{d^{4}}{d x^{4}}\left(x^{2} F(x)\right)\right) \\
& \quad \frac{y^{3} \ln y F(y)}{\left(y+m^{2}\right)^{2}} d y=x^{3} \ln x \frac{d^{3}}{d x^{3}}\left(x^{2} \frac{d^{4}}{d x^{4}}\left(x^{2} F(x)\right)\right)-\left(3 x^{2} \ln x+x^{2}\right) \times \\
& \quad \times \frac{d^{2}}{d x^{2}}\left(x^{2} \frac{d^{4}}{d x^{4}}\left(x^{2} F(x)\right)\right)+(6 x \ln x+5 x) \frac{d}{d x}\left(x^{2} \frac{d^{4}}{d x^{4}}\left(x^{2} F(x)\right)\right)- \\
& \quad-(6 \ln x+11) x^{2} \frac{d^{4}}{d x^{4}}\left(x^{2} F(x)\right)+6 x \frac{d^{3}}{d x^{3}}\left(x^{2} F(x)\right)-6 \frac{d^{2}}{d x^{2}}\left(x^{2} F(x)\right) .
\end{aligned}
$$

Для уравнения (38) следует изменить знак перед $\beta$ и заменить $F(x)$ на $\Psi(x)$.

Благодарности. Работа выполнена при поддержке гранта РФФИ №01-02-16209 и гранта “Университеты России” УР.02.03.028.

\section{Список литературы}

[1] N. N. Bogolubov. Physica (Suppl.). 1960. V. 26. P. 1.

[2] Н. Н. Боголюбов. Квазисредние в задачах статистической механики. Препринт ОИЯИ Д-781. Дубна: ОИЯИ, 1961.

[3] Б. А. Арбузов, А. Н. Тавхелидзе, Р. Н. Фаустов. ДАН СССР. 1961. Т. 139. С. 345.

[4] P. W. Higgs. Phys. Lett. 1964. V. 12. P. 132.

[5] R. S. Chivukula, M. Narain, J. Wormsley. Phys. Rev. D. 2002. V. 66. P. 929.

[6] B. A. Arbuzov. Muon $g-2$ anomaly and extra interaction of composite Higgs in dynamically broken electroweak theory. Preprint NPI MSU 2001-35/675. Moscow: NPI, 2001.

[7] B. A. Arbuzov. Composite $115 \mathrm{GeV}$ Higgs in a dynamically broken electroweak theory. In: Proc. of the IX Conference on Supersymmetry and Unification of Fundamental Interactions. Eds. D. I. Kazakov, A. V. Gladyshev. Singapore: World Scientific, 2002. P. 273.

[8] Н. Н. Боголюбов, Д. В. Ширков. Введение в теорию квантованных полей. М.: Наука, 1973.

[9] А. П. Прудников, Ю. А. Брычков, О. И. Маричев. Интегралы и ряды. Т. 1. Элементарные функции. М.: Наука, 1981.

[10] Б. А. Арбузов, А. Т. Филиппов. ЖЭТФ. 1965. Т. 49. С. 990.

[11] B. A. Arbuzov, A. T. Filippov. Nuovo Cimento. 1965. V. 38. P. 796.

[12] Г. Бейтмен, А. Эрдейи. Высшие трансцендентные функции. Т. 1. Гипергеометрическая функция, функция Лежандра. М.: Наука, 1965.

[13] А. П. Прудников, Ю. А. Брычков, О. И. Маричев. Интегралы и ряды. Т. 3. Дополнительные главы. М.: Наука, 1986.

[14] J. Goldstone. Nuovo Cimento. 1961. V. 19. P. 154. 